\title{
Uso da ostra Crassostrea rhizophorae como filtro biológico para tratamento de efluentes da carcinicultura
}

\section{Use of the oyster Crassostrea rhizophorae as a biological filter for effluent treatment to shrimp farm}

\author{
Karen Figueiredo de Oliveira ${ }^{1}$; Rafael Vieira de Azevedo ${ }^{2}$; \\ Marcelo Cordeiro Pereira ${ }^{3}$; Marcel José Martins dos Santos ${ }^{4}$; \\ João Sérgio Oliveira Carvalho ${ }^{4}$; Luís Gustavo Tavares Braga ${ }^{5 *}$
}

\section{Resumo}

Este estudo teve como objetivo avaliar a capacidade da ostra Crassostrea rhizophorae como filtro biológico para o tratamento de efluentes da carcinicultura e verificar a sua condição microbiológica após processo de filtração. Um total de 1080 ostras foram distribuídas em lanternas de engorda, mantidas em 12 tanques de fibra de vidro $(170 \mathrm{~L})$, em um delineamento inteiramente casualizado, com quatro tratamentos (sem ostras, 60, 120 e 180 ostras) e três repetições. O efluente utilizado foi proveniente do tanque de sedimentação de uma fazenda de produção de camarão. Semanalmente foram analisados: amônio $\left(\mathrm{N}^{-\mathrm{NH}_{4}}{ }^{+}\right)$, ortofosfato $\left(\mathrm{P}_{-} \mathrm{PO}_{4}{ }^{3-}\right)$, total de sólidos suspensos (TSS) e clorofila- $a(\mathrm{Cl}-a)$ do efluente de entrada e saída. Os tratamentos com ostras apresentaram remoção semelhante $(\mathrm{P}>0,05)$ nos teores de $\mathrm{Cl}-a$ e TSS do efluente, sendo superiores $(\mathrm{P}<0,05)$ ao tratamento sem ostras. Não foi observada melhoria na qualidade do efluente após a passagem pelos tanques experimentais em relação ao $\mathrm{N}-\mathrm{NH}_{4}{ }^{+}$. Em relação ao $\mathrm{P}_{-} \mathrm{PO}_{4}{ }^{3-}$, todos os tratamentos reduziram as concentrações desse nutriente de forma semelhante $(\mathrm{P}>0,05)$. Não foram observadas diferenças $(\mathrm{P}>0,05)$ para os valores de coliformes totais e de cobre, independentemente da densidade de estocagem. Nas amostras analisadas não foram encontrados coliformes fecais e Salmonella spp. As ostras melhoram a qualidade da água, exceto $\mathrm{N}-\mathrm{NH}_{4}^{+}$, e não apresentam contaminantes que inviabilizem seu consumo.

Palavras-chave: Aquicultura integrada, Litopenaeus vannamei, microbiologia

\begin{abstract}
This study aimed to evaluate the biofiltration capacity of oyster Crassostrea rhizophorae on effluents of shrimp and check their microbiological condition after filtering process. 1.080 oysters were distributed in lanterns kept 12 fiberglass tanks $(170 \mathrm{~L})$ in a completely randomized design with four treatments (without oysters, 60, 120 and 180 oysters) and three repetitions. It was used the effluent from the sedimentation tank. Weekly were analyzed: ammonia nitrogen $\left(\mathrm{N}^{-\mathrm{NH}_{4}+}\right)$, orthophosphate $\left(\mathrm{P}^{-} \mathrm{PO}_{4}{ }^{3-}\right)$, total suspended solids (TSS) and chlorophyll- $a(\mathrm{Cl}-a)$ of the input and output effluent. The treatments
\end{abstract}

\footnotetext{
${ }^{1}$ Discente do Curso de Doutorado do Programa de Pós-Graduação em Ecologia e Recursos Naturais, Universidade Federal do Ceará, UFC, Fortaleza, CE. E-mail: karenbiologa@hotmail.com

${ }^{2}$ Discente do Curso de Doutorado do Programa de Pós-Graduação em Ciência Animal, Universidade Estadual do Norte Fluminense Darcy Ribeiro, UENF, Campos dos Goytacazes, RJ. E-mail: azevedorv84@ gmail.com

${ }^{3}$ Prof. do Centro de Ensino Superior de Ilhéus, BA. E-mail: maquicultura@yahoo.com.br

${ }^{4}$ Pesquisadores do Laboratório de Nutrição e Alimentação de Peixes, AQUANUT. Universidade Estadual de Santa Cruz, UESC, Ilhéus, BA. E-mail: marcel_martins_santos@hotmail.com; joaosoc@gmail.com

${ }^{5}$ Prof. Dr. em Zootecnia, Dept ${ }^{\mathrm{o}}$ de Ciências Agrárias e Ambientais da UESC, Ilhéus, BA. E-mail: lgtbraga@gmail.com

* Autor para correspondência
} 
with oysters had similar removal effect $(\mathrm{P}>0.05)$ in the levels of Cl- $a$ and TSS effluent and were higher $(\mathrm{P}<0.05)$ to the treatment without oysters. There was no improvement in the quality of the effluent after passing through the experimental tanks to $\mathrm{N}_{-} \mathrm{NH}_{4}^{+}$. In relation to $\mathrm{P}_{-} \mathrm{PO}_{4}{ }^{3-}$, all treatments reduced $(\mathrm{P}>0.05)$ the concentrations of this nutrient similarly. There were no statistical differences $(\mathrm{P}>0.05)$ for the values of total coliform and copper, regardless of stocking density. The samples analyzed were not

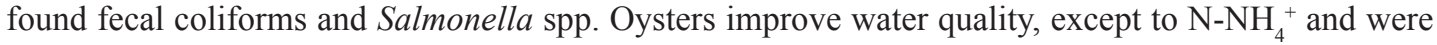
not found organisms that prohibit its consumption.

Key words: Integrated aquaculture, Litopenaeus vannamei, microbiology

\section{Introdução}

Aquicultura é uma das alternativas mais promissoras para o fornecimento de alimento de alto valor nutritivo, impulsionada pelo crescente déficit entre a quantidade de pescado capturado e a elevada demanda do mercado consumidor (CAMARGO; POUEY, 2005; DUMBAULD; RUESINKB; RUMRILLC, 2009).

No Brasil, o cultivo de crustáceos foi impulsionado pela introdução do camarão branco do Pacífico Litopenaeus vannamei. Seu desempenho zootécnico $\left(5.458 \mathrm{~kg} \mathrm{ha}^{-1} \mathrm{ano}^{-1}\right)$ o levou ao primeiro lugar em produtividade mundial por volta do ano de 2004 (MENDES et al., 2006), sendo a região Nordeste responsável por cerca de $97 \%$ da produção nacional de camarões (MPA, 2012).

Entretanto, em 2004, o setor sofreu forte crise desencadeada, principalmente, pela disseminação do vírus da mancha branca em diversas fazendas de camarão, a ação antidumping dos Estados Unidos e a instabilidade do dólar (ARAÚJO; OKINO, 2009). Em 2010, a aquicultura marinha contribuiu com $17,7 \%$ da produção aquícola nacional correspondendo a 69.422 t, sendo $97 \%$ desta produção oriunda da carcinicultura (MPA, 2012). Apesar de a carcinicultura apresentar grande potencial econômico, tecnologias não apropriadas podem ocasionar uma série de impactos ambientais nos ecossistemas naturais. A adubação contínua, renovação constante de água e arraçoamento em excesso acabam por gerar um efluente rico em nutrientes. Estes são liberados diretamente, e muitas vezes, sem qualquer tratamento prévio, nos ambientes adjacentes aos tanques de cultivo. Segundo Jones, Dennison e
Preston (2001) efluentes de viveiros de camarão normalmente contêm elevadas concentrações de nutrientes dissolvidos e partículas em suspensão, quando comparados a seus afluentes.

O uso de lagoas de sedimentação em países produtores de camarão tem sido uma forma de tratar os efluentes. Estas lagoas são eficazes na redução de descargas de partículas suspensas, porém não são eficientes quanto à redução das concentrações dos nutrientes dissolvidos (JACKSON et al., 2003). Nesse sentido, a integração de organismos potencialmente filtradores pode aumentar a eficiência na redução do impacto causado pelos efluentes. As tentativas para melhorar a qualidade de água de efluentes utilizando bivalves vêm sendo estudadas como parte do desenvolvimento de uma aquicultura integrada, que além de melhorar a qualidade da água, pode ainda resultar em incremento econômico (LEFEBVRE; BARILLÉ; CLERC, 2001).

Dentre os moluscos bivalves cultiváveis, destacam-se as ostras (JONES; PRESTON, 1999), que possuem hábito alimentar filtrador, alimentandose de partículas em suspensão na água. Através do fluxo de água, o alimento passa através das cavidades do manto, pelas brânquias, que funcionam como um filtro e concentram partículas orgânicas, algas microscópicas e organismos planctônicos servindo de alimento para o animal (WARD, 1996).

Porém, durante o processo de filtração, as ostras podem absorver toxinas, poluentes químicos e biológicos, inclusive microorganismos e metais pesados presentes na água. O cobre é um dos importantes contaminantes emitidos pelos efluentes 
da carcinicultura. O cultivo intensivo de Litopenaeus vannamei pode funcionar como acumulador no sedimento e emissor de cobre nos efluentes, provenientes da alimentação, outros insumos e exúvias (LACERDA; SANTOS; LOPES, 2009).

O objetivo deste trabalho foi avaliar a capacidade da ostra Crassostrea rhizophorae como filtro biológico para o tratamento de efluentes da carcinicultura e verificar a sua condição microbiológica após processo de filtração.

\section{Material e Métodos}

O experimento foi desenvolvido na Fazenda Maricanes, localizada no município de Canavieiras, sul do estado da Bahia (1540’30'’S e 38 56'50”W), durante quatro semanas, entre os meses de maio e julho de 2010.

A fazenda possui onze viveiros escavados, totalizando 43 hectares de lâmina d'água e duas lagoas de sedimentação, com $5000 \mathrm{~m}^{2}$ de área e $2 \mathrm{~m}$ de profundidade cada uma. No momento da execução do experimento, apenas sete viveiros estavam povoados com o camarão Litopenaeus vannamei, com 30 dias de cultivo, em uma densidade de 10 indivíduos por $\mathrm{m}^{2}$, alimentados duas vezes ao dia, com ração comercial peletizada contendo $36 \%$ de proteína bruta, $7 \%$ de extrato etéreo, $9 \%$ de fibras e $12 \%$ de matéria mineral (níveis de garantia fornecidos pelo fabricante).

Para o tratamento dos efluentes foi montado um sistema constituído de 12 tanques experimentais de fibra de vidro com capacidade individual de $170 \mathrm{~L}$, instalados a cinco metros da margem da lagoa de sedimentação da fazenda. A estrutura foi abastecida com um sistema de fluxo contínuo de água proveniente da lagoa de sedimentação com auxílio de uma bomba d'água de $1 \mathrm{cv}$.

O efluente foi distribuído de forma independente em cada tanque por meio de tubulação de PVC, onde a entrada se dava pela superfície, com vazão constante e controlada por registro de $15 \mathrm{~mL} \mathrm{~s}^{-1}$ e a saída por sistema "tipo" monge.

Foram utilizados 1080 exemplares adultos da espécie Crassostrea rhizophorae com altura média de $10 \pm 0,4 \mathrm{~cm}$, obtidos de produção comercial na Baía de Camamu, Bahia (1340'02'S e $38^{\circ} 55^{\prime} 08^{\prime}$ 'O). As ostras foram distribuídas em lanternas de engorda, de formato cilíndrico com $30 \mathrm{~cm}$ de diâmetro e altura de $120 \mathrm{~cm}$ com três andares, de acordo com o tratamento: controle (sem ostras), 60 ostras (20 andar $\left.^{-1}\right), 120$ ostras (40 ostras andar ${ }^{-1}$ ) e 180 ostras (60 ostras andar $\left.{ }^{-1}\right)$. O delineamento experimental utilizado foi o inteiramente casualizado, com quatro tratamentos e três repetições. Após a alocação das ostras, as lanternas foram colocadas nos tanques mantendo-se uma distância de $15 \mathrm{~cm}$ do fundo.

Semanalmente, entre 9:00h e 10:00h, foram coletadas amostras de 2 L do efluente de entrada e saída de cada tanque experimental, acondicionadas e mantidas refrigeradas em isopor com gelo e transportados para Universidade Estadual de Santa Cruz (UESC), Ilhéus, Bahia, para posteriores análises. No próprio local de coleta foram analisados os seguintes parâmetros: oxigênio dissolvido utilizando um oxímetro (YSI modelo 55-12FT), pH e temperatura através de um multiparâmetro (YSY modelo 63-10FT) e salinidade, através de refratômetro óptico manual (Atago S/Mill).

No laboratório de Oceanografia Química e Monitoramento Ambiental foram analisados: amônio $\left(\mathrm{N}-\mathrm{NH}_{4}^{+}\right)$, ortofosfato $\left(\mathrm{P}-\mathrm{PO}_{4}^{-3}\right)$, clorofila- $a$ (Cl-a) e total de sólidos suspensos (TSS). A determinação de $\mathrm{N}_{-} \mathrm{NH}_{4}^{+}$e $\mathrm{P}_{-} \mathrm{PO}_{4}^{3-}$ foi realizada em espectrofotômetro (LAMBDA EZ 201). Para a determinação da $\mathrm{Cl}-a$ foi utilizado acetona $80 \%$ para extração seguindo a metodologia de Jeffrey e Humphrey (1975) e posterior leitura em espectrofotômetro. A mensuração do TSS após processo de filtragem a vácuo foi realizada segundo APHA (1998), sendo obtido o peso do material particulado em suspensão (mg L-1 $)$.

A remoção de nutrientes [(concentração no efluente de entrada - concentração no efluente 
após os tanques experimentais) / concentração no efluente de entrada] x 100 foi calculada, para cada tratamento, semanalmente.

Para análise da qualidade sanitária das ostras, três exemplares de cada unidade experimental foram coletados e acondicionados em sacos plásticos em caixas de isopor com gelo, para manterem temperatura inferior a $10^{\circ} \mathrm{C}$ durante o transporte até o laboratório para análise da presença de Salmonella, coliformes totais, fecais e metal pesado (cobre).

O processamento das ostras foi realizado no Laboratório de Monitoramento Ambiental. Para detecção de coliformes fecais e totais foram separadas partes moles e valvas das ostras, de forma que não houvesse contaminação. Posteriormente cada amostra foi triturada e diluída em água destilada em série de $10^{-1}, 10^{-2}$ e $10^{-3}$. Com auxílio de bomba a vácuo, foi filtrado $1 \mathrm{~mL}$ de cada amostra através de uma membrana filtrante de $47 \mathrm{~mm}$ de diâmetro e $0,45 \mu \mathrm{m}$ de porosidade estéril. Posteriormente essas membranas foram colocadas sobre a superfície da placa de Petri contendo o meio de cultura Chromocult Coliform Agar, incubadas a $37^{\circ} \mathrm{C}$ por 24 horas em estufa para posterior contagem de unidades formadoras de colônias (UFC).

A detecção de Salmonella foi realizada segundo a técnica dos Tubos Múltiplos com três séries de quatro tubos (APHA, 1998), constando de um teste presuntivo e três testes confirmativos.

Para análise de cobre as ostras foram medidas, lavadas e abertas e as partes moles foram acondicionadas em bandejas de alumínio, pesadas e mantidas em estufa a $60^{\circ} \mathrm{C}$ por 24 horas. Após esse período, as amostras foram retiradas, novamente pesadas, acondicionadas em frasco de vidro e enviadas ao laboratório de Biogeoquímica Costeira/ LABOMAR da Universidade Federal do Ceará. As determinações de cobre foram efetuadas por espectrofotometria de absorção atômica de chama convencional, utilizando o espectrofotômetro de absorção atômica (AA-6200 Shimadzu), com média de limite de detecção de $0,14 \mu \mathrm{g} \mathrm{ml}^{-1}$ para cobre. Os resultados foram expressos em $\mu \mathrm{g} \mathrm{g}^{-1}$ de peso seco. Para facilitar a comparação com as concentrações máximas permitidas pelo Ministério da Saúde, expressas em peso úmido, considerouse a concentração de cobre obtida em peso seco multiplicado pelo percentual de umidade.

Os dados de remoção de nutrientes e de análise microbiológica das ostras obtidos ao final do experimento foram submetidos à análise de variância em nível de 5\% de significância. Em caso de diferenças, aplicou-se o teste de Tukey, utilizando-se o programa Statistical Analysis System 9.0 (SAS).

\section{Resultados e Discussão}

No decorrer do período experimental, os valores dos parâmetros de temperatura, oxigênio dissolvido e salinidade do efluente de entrada do sistema apresentaram comportamento semelhante, observando-se queda nos valores desses parâmetros na segunda semana em relação à primeira, provavelmente ocasionada pelas chuvas ocorridas, a partir da qual os valores se elevaram. $\mathrm{O}$ pH apresentou comportamento decrescente durante todo o período experimental (Figura 1).

As condições ambientais influenciam diretamente a taxa de filtração dos bivalves (JONES; PERSTON; DENNISON, 2002). Neste estudo os valores de temperatura e $\mathrm{pH}$ estiveram dentro da faixa recomendada para o cultivo da ostra Crassostrea rhizophorae, entre de $24,9^{\circ} \mathrm{C}$ a $28,2^{\circ} \mathrm{C}$ e 6,3 a 7,5 respectivamente, corroborando os dados de Frias e Rodrigues (1991). Os valores de oxigênio dissolvido apresentaram variações entre 2,5 a 3,2 $\mathrm{mg} \mathrm{L}^{-1}$ com uma queda nas concentrações na segunda semana de cultivo (1,2 $\left.\mathrm{mg} \mathrm{L}^{-1}\right)$. Assim, baseado no estudo de Reyes (1995), exceto na segunda semana, provavelmente os valores de oxigênio dissolvido não prejudicaram o desenvolvimento e a alimentação das ostras. Os valores de salinidade (menores que $10 \mathrm{ppt}$ durante todo o período experimental) apresentaram- 
se abaixo do recomendado para o cultivo de ostras dessa espécie. Segundo Guimarães et al. (2008), C. rhizophorae cultivada em salinidades compreendidas entre 20 e 30 ppt apresenta maior eficiência na taxa de alimentação, porém, não foi observada mortalidade dos organismos no inicio do experimento, mostrando a ampla faixa de adaptação dessa espécie à baixas concentrações salinas.

Figura 1. Variação semanal das variáveis físicas e químicas do efluente de entrada no sistema experimental.
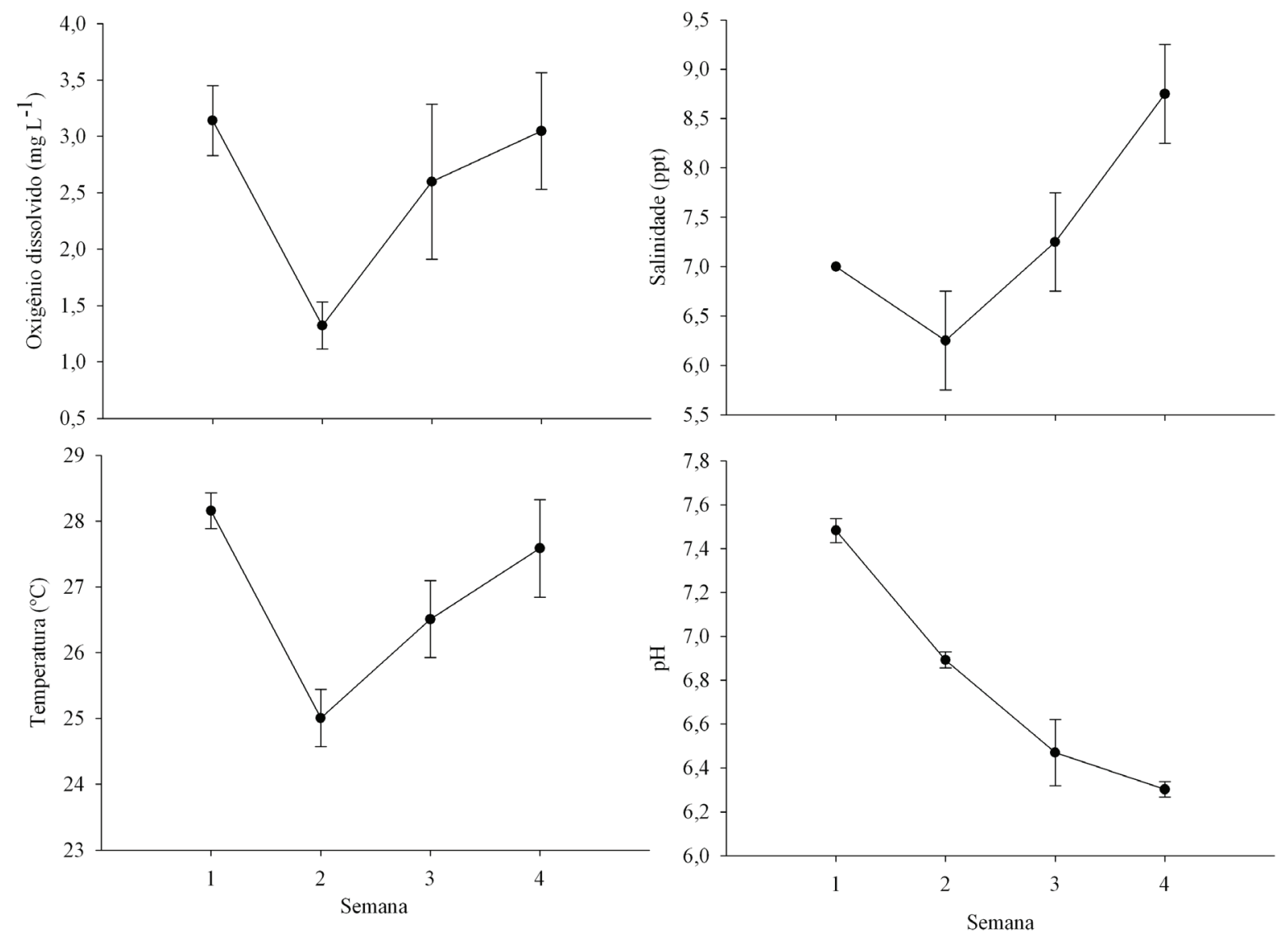

Fonte: Elaboração dos autores.

Ao longo do período experimental, os tratamentos com ostras, independentemente da densidade, apresentaram remoção semelhante ( $\mathrm{P}>0,05)$ para os teores de $\mathrm{Cl}-a$ e TSS dos efluentes, diferindo significativamente do tratamento controle (sem ostras) $(\mathrm{P}<0,05)$ (Figura 2 e Tabela 1). Tal fato demonstra a eficiência da remoção destes itens, pela introdução das ostras no sistema, corroborando os trabalhos de Dame (1993); Soto e Mena (1999), que observaram grande redução de fitoplancton na coluna d'água. Já em experimento em laboratório, Jones, Dennison e Perston (2001) e Ramos, Vinatea e Costa (2008), utilizando as ostras Saccostrea commercialis e C. rhizophorae, respectivamente, observaram remoção acima de 70\% nas concentrações da $\mathrm{Cl}-a$ de efluentes provindos da carcinicultura, sendo esta a mesma situação ocorrida neste experimento. 
Figura 2. Variação semanal de nutrientes dos efluentes da entrada dos tanques e após a passagem pelos tanques experimentais com $0,60,120$ e 180 ostras.
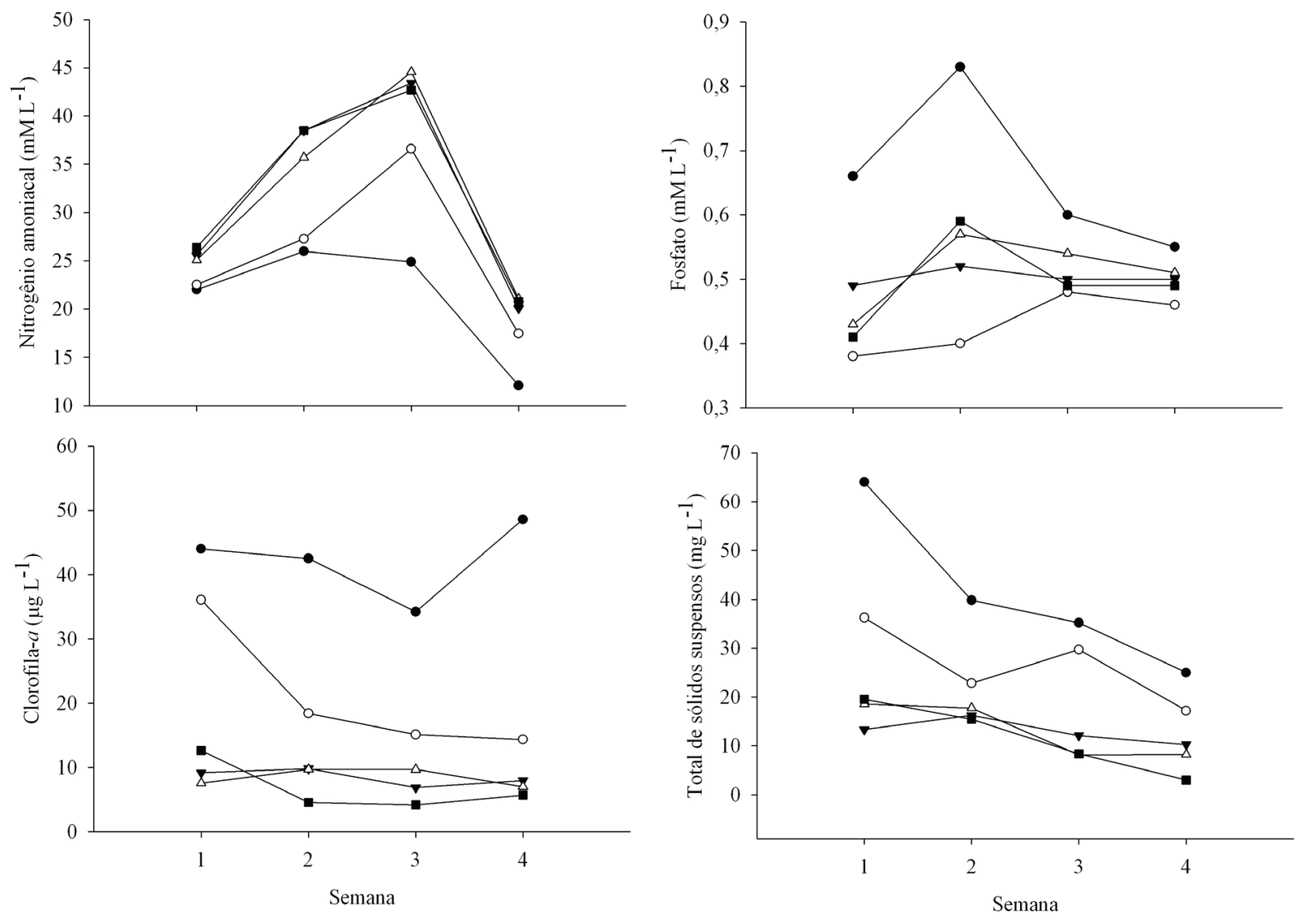

$\longrightarrow$ Entrada $\longrightarrow 0$ ostra $\longrightarrow 60$ ostras $\quad \longrightarrow 120$ ostras $\longrightarrow 180$ ostras

Fonte: Elaboração dos autores.

Segundo Jones, Perston e Dennison (2002), as ostras removem grandes quantidades de fitoplâncton, bactérias e sólidos suspensos na coluna d'água, selecionando as partículas por tamanho, peso e composição química, consumindo preferencialmente matéria orgânica em detrimento da inorgânica, o que pode explicar a grande eficiência na remoção de clorofila- $a$ neste estudo.

A remoção de sólidos suspensos tem se mostrado eficiente pelo processo de filtração através do uso de bivalves (JONES; DENNISON; PERSTON, 2001; JONES; PERSTON; DENNISON, 2002). Ramos et al. (2009) observaram redução de 70,6\% de sólidos suspensos de efluente da carcinicultura utilizando a ostra $C$. rhizophorae em condições laboratoriais com animais entre 76 e $80 \mathrm{~g}$, sendo superiores aos observados nesse experimento. Estas diferenças observadas podem estar relacionadas ao tamanho dos animais, tempo de residência e salinidade da água. Segundo Madrigal et al. (1985) a taxa de filtração ótima para $C$. rhizophorae a uma temperatura de $28^{\circ} \mathrm{C}$ é alcançada com uma salinidade de $25 \mathrm{ppt}$, valor 2,8 vezes superior ao observado nesse experimento (máximo de 9 ppt). Ainda, os experimentos comparados foram realizados em escala laboratorial, não sujeitando às ostras as alterações na qualidade da água (efluente), que em ambiente natural pode sofrer grande variação, devido não só a características como tempo de cultivo e densidade de estocagem dos 
animais, como a alterações provocadas pelo clima e precipitações, que podem influenciar na maior ou menor remoção de nutrientes (COSTANZO; O’DONOHUE; DENNISON, 2004).

Tabela 1. Porcentagem semanal de remoção de nutrientes de acordo com o tratamento (média \pm desvio padrão)

\begin{tabular}{|c|c|c|c|c|}
\hline \multirow{2}{*}{ Tratamento } & \multicolumn{4}{|c|}{ Parâmetro ${ }^{1}$} \\
\hline & $\mathrm{Cl}-a$ & TSS & $\mathrm{N}^{-\mathrm{NH}_{4}^{+}}$ & $\mathrm{P}_{-} \mathrm{PO}_{4}^{3-}$ \\
\hline \multicolumn{5}{|l|}{ Semana 1} \\
\hline Controle & $18,01 \pm 1,21^{\mathrm{b}}$ & $43,39 \pm 2,76^{b}$ & $\uparrow 2,36 \pm 0,42^{\mathrm{b}}$ & $42,42 \pm 10,02^{\mathrm{a}}$ \\
\hline 60 ostras & $79,15 \pm 9,12^{\mathrm{a}}$ & $79,15 \pm 12,91^{\mathrm{a}}$ & $\uparrow 16,14 \pm 2,14^{\mathrm{a}}$ & $25,76 \pm 4,23^{\mathrm{a}}$ \\
\hline 120 ostras & $82,82 \pm 12,21^{\mathrm{a}}$ & $70,95 \pm 6,42^{\mathrm{a}}$ & $\uparrow 14,05 \pm 2,87^{\mathrm{a}}$ & $34,85 \pm 4,11^{\mathrm{a}}$ \\
\hline 180 ostras & $71,23 \pm 9,78^{\mathrm{a}}$ & $69,50 \pm 5,04^{\mathrm{a}}$ & $\uparrow 19,91 \pm 2,10^{\mathrm{a}}$ & $37,88 \pm 6,45^{\mathrm{a}}$ \\
\hline \multicolumn{5}{|l|}{ Semana 2} \\
\hline Controle & $56,61 \pm 3,23^{b}$ & $42,68 \pm 4,63^{b}$ & $\uparrow 4,96 \pm 0,76^{\mathrm{b}}$ & $51,81 \pm 9,44^{\mathrm{a}}$ \\
\hline 60 ostras & $76,85 \pm 8,12^{\mathrm{a}}$ & $59,21 \pm 3,10^{\mathrm{a}}$ & $\uparrow 48,21 \pm 7,12^{\mathrm{a}}$ & $37,35 \pm 3,79^{\mathrm{a}}$ \\
\hline 120 ostras & $77,15 \pm 9,02^{\mathrm{a}}$ & $55,46 \pm 2,88^{\mathrm{a}}$ & $\uparrow 37,39 \pm 3,99^{\mathrm{a}}$ & $31,33 \pm 3,77^{\mathrm{a}}$ \\
\hline 180 ostras & $89,20 \pm 13,21^{\mathrm{a}}$ & $61,08 \pm 8,11^{\mathrm{a}}$ & $\uparrow 48,13 \pm 5,12^{\mathrm{a}}$ & $28,92 \pm 3,94^{\mathrm{a}}$ \\
\hline \multicolumn{5}{|l|}{ Semana 3} \\
\hline Controle & $55,82 \pm 7,45^{b}$ & $15,56 \pm 3,12^{b}$ & $\uparrow 47,20 \pm 3,87^{b}$ & $20,00 \pm 3,30^{\mathrm{a}}$ \\
\hline 60 ostras & $79,85 \pm 9,66^{\mathrm{a}}$ & $65,55 \pm 8,54^{\mathrm{a}}$ & $\uparrow 74,62 \pm 7,87^{\mathrm{a}}$ & $16,66 \pm 3,76^{\mathrm{a}}$ \\
\hline 120 ostras & $71,67 \pm 12,01^{\mathrm{a}}$ & $76,71 \pm 5,12^{\mathrm{a}}$ & $\uparrow 79,17 \pm 5,99^{\mathrm{a}}$ & $10,00 \pm 1,23^{\mathrm{a}}$ \\
\hline 180 ostras & $87,69 \pm 12,21^{\mathrm{a}}$ & $76,14 \pm 8,73^{\mathrm{a}}$ & $\uparrow 71,65 \pm 9,76^{\mathrm{a}}$ & $18,33 \pm 1,77^{\mathrm{a}}$ \\
\hline \multicolumn{5}{|l|}{ Semana 4} \\
\hline Controle & $70,45 \pm 8,22^{b}$ & $31,26 \pm 3,72^{b}$ & $\uparrow 44,69 \pm 2,23^{b}$ & $16,36 \pm 2,87^{\mathrm{a}}$ \\
\hline 60 ostras & $83,56 \pm 7,12^{\mathrm{a}}$ & $58,95 \pm 5,44^{\mathrm{a}}$ & $\uparrow 66,33 \pm 5,87^{\mathrm{a}}$ & $9,09 \pm 1,01^{\mathrm{a}}$ \\
\hline 120 ostras & $85,56 \pm 6,23^{\mathrm{a}}$ & $66,95 \pm 7,84^{\mathrm{a}}$ & $\uparrow 74,46 \pm 10,08^{\mathrm{a}}$ & $7,28 \pm 1,04^{\mathrm{a}}$ \\
\hline 180 ostras & $88,18 \pm 12,97^{\mathrm{a}}$ & $88,01 \pm 5,77^{\mathrm{a}}$ & $\uparrow 72,30 \pm 5,33^{\mathrm{a}}$ & $10,90 \pm 0,88^{\mathrm{a}}$ \\
\hline
\end{tabular}

Médias, nas colunas, para cada semana, seguidas por letras diferentes diferem estatisticamente pelo teste de Tukey, a $5 \%$ de probabilidade.

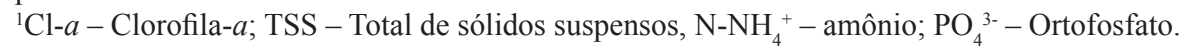

$\uparrow-$ aumento na concentração do nutriente no efluente.

Fonte: Elaboração dos autores.

Não foi detectada melhoria na qualidade do efluente após a passagem pelos tanques experimentais em relação ao $\mathrm{N}_{-} \mathrm{NH}_{4}^{+}$, observandose elevação nos teores deste nutriente, sendo esta significativamente superior $(\mathrm{P}<0,05)$ nos tanques com ostras, independentemente da densidade, em relação aos tanques sem ostras. Semelhante aos resultados neste estudo, Jones, Dennison e Perston (2001), Gurjão (2004) e Ramos, Vinatea e Costa, (2008) observaram aumento na concentração de $\mathrm{N}-\mathrm{NH}_{4}^{+}$em tratamentos com ostras. Esse resultado era esperado, uma vez que a maioria dos organismos aquáticos libera amônia como excreta nitrogenada (RUPPERT; BARNES, 1996). Jones, Dennison e Perston (2001) observaram taxa de liberação de amônia por $S$. commercialis de $0,52 \mu \mathrm{mol} \mathrm{h} \mathrm{h}^{-1}$.

Em relação ao $\mathrm{P}_{-} \mathrm{PO}_{4}^{3-}$ do efluente, foram observados reduções nas concentrações deste nutriente em relação ao efluente de entrada, porém, entre os tratamentos não foram observadas diferenças significativas $(\mathrm{P}>0,05)$ reduzindo as concentrações desse nutriente de forma semelhante ao tratamento controle. Cavalcante-Júnior et al. (2005) e Ramos et al. (2010) observaram remoção de, respectivamente, 
$56,1 \%$ e $6,5 \%$ nos teores de $\mathrm{P}-\mathrm{PO}_{4}^{3-}$ pelo processo de filtração de ostras. Neste experimento, além da redução pelo processo de filtração, bactérias heterotróficas presentes nos tanques podem ter convertido o fósforo em biomassa bacteriana (EBELING; TIMMONS; BISOGNI, 2006), auxiliando na remoção do fosfato.
$\mathrm{Na}$ avaliação microbiológica das ostras, não foram observadas diferenças estatísticas $(\mathrm{P}>0,05)$ para os valores de coliformes totais e de metal traço (cobre), independentemente da densidade de estocagem, bem como não foram encontrados coliformes fecais e Salmonella spp (Tabela 2).

Tabela 2. Valor médio e desvio padrão de coliformes totais, coliformes fecais, cobre e avaliação de Salmonella spp. em ostras de acordo com o tratamento.

\begin{tabular}{ccccc}
\hline Tratamento & $\begin{array}{c}\text { Coliformes totais } \\
\left(\mathrm{UFC} 100 \mathrm{~mL}^{-1}\right)\end{array}$ & $\begin{array}{c}\text { Coliformes fecais } \\
\left(\mathrm{UFC} 100 \mathrm{~mL}^{-1}\right)\end{array}$ & Salmonella spp. & $\begin{array}{c}\text { Cobre } \\
\left(\mu \mathrm{g} \mathrm{g}^{-1}\right)^{(1)}\end{array}$ \\
\hline 60 ostras & $10133,3 \pm 1715,6$ & 0 & Ausente & $15,6 \pm 1,7$ \\
120 ostras & $15800,0 \pm 11407,7$ & 0 & Ausente & $16,6 \pm 6,4$ \\
180 ostras & $16400,0 \pm 2306,5$ & 0 & Ausente & $13,7 \pm 5,3$ \\
\hline
\end{tabular}

${ }^{(1)}$ Valores expressos com base na matéria úmida.

Fonte: Elaboração dos autores.

Embora o uso de moluscos como biofiltros de efluentes de carcinicultura apresente grande eficiência, o mecanismo de filtração permite $o$ acúmulo de grande quantidade de microorganismos e, consequentemente, o armazenamento de rica flora bacteriana, podendo agir como portadoras passivas de agentes patogênicos ao homem, quando mantidas em águas poluídas (BURKHARDT; CALCI, 2000), uma vez que as ostras são geralmente consumidas in natura sem prévio cozimento.

A Resolução vigente $\mathrm{n}^{\circ} 12$ da Agência Nacional de Vigilância Sanitária (ANVISA) não oferece critérios para a avaliação de moluscos consumidos in natura, contemplando somente moluscos bivalves cozidos, industrializados resfriados ou congelados, somente contemplando a contagem de UFC g ${ }^{-1}$ de Estafilococos coagulasepositivo e presença de Salmonella spp. (BRASIL, 2001). Muitos países que comercializam ostras desenvolveram um conjunto de normas próprias, baseadas em análises microbiológicas da água de seu cultivo e/ou da sua carne. A maioria dessas normas está baseada na pesquisa de coliformes fecais (MACHADO et al., 2001).

O cobre é um metal traço essencial para moluscos, pois participa da constituição do pigmento respiratório hemocianina (RAINBOW et al., 1999). Assim, esse metal é requerido em certas concentrações para o metabolismo do animal. Entretanto, concentrações elevadas têm o potencial tóxico para os animais e consumidores. As concentrações de cobre obtidas nas análises do tecido das ostras nesse estudo apresentaram valor médio de $15,3 \mu \mathrm{g} \mathrm{g}^{-1}$, permanecendo abaixo do valor máximo $\left(30 \mu \mathrm{g} \mathrm{g}^{-1}\right)$ permitido pela legislação brasileira no Decreto $\mathrm{n}^{0} 55871$, de 26 de março de 1965, que determina limites máximos de tolerância para contaminantes inorgânicos (BRASIL, 1965), portanto seu consumo não caracteriza risco à saúde humana.

De acordo com os dados obtidos neste estudo, a utilização da ostra $C$. rhizophorae como biofiltro para efluentes da carcinicultura pode reduzir as partículas em suspensão e o fitoplâncton, o que gera um efluente de melhor qualidade para o descarte no ambiente. Ressalta-se que as ostras não são efetivas em remover o nitrogênio amoniacal do efluente devido ao seu metabolismo. No entanto, estudos avaliando a eficiência de macroalgas (RAMOS et al., 2008) e macrófitas (HENRY-SILVA; CAMARGO, 2008) no 
tratamento de efluentes da carcinicultura, mostraram que estas podem reduzir de forma significativa os valores de nitrogênio amoniacal. Assim, com base nesses resultados, recomenda-se a consorciação de ostras com macroalgas e macrófitas para maior eficiência no tratamento de efluentes da carcinicultura.

\section{Conclusão}

A utilização de ostras como biofiltros de efluentes da aquicultura reduz as partículas de sólidos em suspenção e também o fitoplâncton, mas não tem mesmo efeito para o nitrogênio amoniacal. E na análise microbiológica das ostras, não foram encontrados organismos que inviabilizam seu consumo.

\section{Agradecimentos}

Os autores agradecem a Coordenação de Aperfeiçoamento de Pessoal de Nível Superior (CAPES), a Fundação de Amparo à Pesquisa do Estado da Bahia (FAPESB), ao Instituto Nacional de Ciência e Tecnologia de Transferência de Materiais Continente-Oceano (INCT-TMCOcean) e a Fazenda Maricanes pelo apoio no desenvolvimento do estudo e a Dra. Daniela Mariano Lopes da Silva (UESC) pelo apoio na realização das análises dos parâmetros de qualidade de água.

\section{Referências}

AMERICAN PUBLIC HEALTH ASSOCIATION APHA. Standard Methods for the examination of water and wasterwater. 20. ed. New York: Amercian Public Health Association, 1998. 1325 p.

ARAÚJO, M. A. D.; OKINO, M. Y. F. Qualidade dos empregos da carcinicultura na praia de Barreta/RN. Organizações Rurais \& Agroindustriais, Lavras, v. 11, n. 1, p. 140-156, 2009.

BRASIL. ANVISA - AGÊNCIA NACIONAL DE VIGILÂNCIA SANITÁRIA. Regulamento técnico sobre os padrões microbiológicos para alimentos. Resolução $\mathrm{n}^{\mathrm{o}}$ 12, de 2 de janeiro de 2001. Diário Oficial [da] República Federativa do Brasil, Brasília, DF, 10 jan. 2001. Seção 1, p. 48.
Ministério da Saúde. Decreto $\mathrm{n}^{\circ} 55871$, de 26 de março de 1965. Modifica o decreto $n^{\circ} 50040$, de 24 de janeiro de 1961, referente às normas reguladoras do emprego de aditivos para alimentos, alterado pelo Decreto $n^{\circ}$ 691, de 13 de março de 1962. Diário Oficial [da] República Federativa do Brasil, Brasília, DF, 9 abr. 1965. Seção 1, p. 3610.

BURKHARDT, W.; CALCI, K. R. Selective accumulation may account for shellfish-associated viral illness. Applied and Environmental. Microbiology, Washington, v. 66, n. 4, p. 1375-1378, 2000.

CAMARGO, S. G. O.; POUEY, J. L. O. F. Aquicultura - um mercado em expansão. Revista Brasileira de Agrociência, Pelotas, v. 11, n. 4, p. 393-396, 2005.

CAVALCANTE-JÚNIOR, V.; ANDRADE, L. N.; BEZERRA, L. N.; GURJÃO, L. M.; FARIAS, W. R. L. Reúso de água em um sistema integrado com peixes, sedimentação, ostras e macroalgas. Revista Brasileira de Engenharia Agrícola e Ambiental, Campina Grande, v. 9 , p. 118-122, 2005. Suplemento.

COSTANZO, S. D.; O’DONOHUE, M. J.; DENNISON, W. C. Assessing the influence and distribution of shrimp pond effluent in a tidal mangrove creek in north-east Australia. Marine Pollution Bulletin, Philadelphia, v. 48, n. 1, p. 514-525, 2004.

DAME, R. F. Bivalve filter feeders in estuarine and coastal ecosystem processes. Berlin: Springer, 1993. 579 p.

DUMBAULD, B. R.; RUESINKB, J. L.; RUMRILLC, S. S. The ecological role of bivalve shellfish aquaculture in the estuarine environment: a review with application to oyster and clam culture in West Coast (USA) estuaries. Aquaculture, Amsterdam, v. 290, n. 3-4, p. 196-223, 2009.

EBELING, J. M.; TIMMONS, M. B.; BISOGNI, J. J. Engineering analysis of the stoichiometry of photoautotrophic, autotrophic, and heterotrophic removal of ammonia-nitrogen in aquaculture systems. Aquaculture, Amsterdam, v. 257, n. 1, p. 346-358, 2006.

FRIAS, J. A.; RODRIGUEZ, R. Oyster culture in Cuba: current state, techniques and industry organization. In: NEWKIRK, G. F.; FIELD, B. A. Oyster culture. Halifax: IDRC, 1991. p. 51-74.

GUIMARÃES, I. M.; ANTONIO, I. G.; PEIXOTO, S.; OLIVEIRA, A. Salinity influence on the survival of mangrove oyster Crassostrea rhizophorae. Arquivos de Ciência do Mar, Fortaleza, v. 41, n. 1, p. 118-122, 2008.

GURJÃO, L. M. O. Uso integrado de sedimentação, ostras e macroalgas para o tratamento de efluentes de carcinicultura. 2004. Dissertação (Mestrado em 
Engenharia de Pesca) - Universidade Federal do Ceará, Fortaleza.

HENRY-SILVA, G. G.; CAMARGO, A. F. M. Tratamento de efluentes de carcinicultura por macrófitas aquáticas flutuantes. Revista Brasileira de Zootecnia, Viçosa, v. 37, n. 2. p. 181-188, 2008.

JACKSON, C. J.; PRESTON, N.; BURFORD, M. A.; THOMPSON, P. J. Managing the development of sustainable shrimp farming in Australian: the role of sedimentation ponds in treatment of farm discharge water. Aquaculture, Amsterdam, v. 226, n. 1-4, p. 23-34, 2003.

JEFFREY, S. W.; HUMPHREY, G. F. New spectrophotometric equation for determining chlorophyll a, b, c1 and c2. Biochemical Physiology Pflanzen, v. 167, n. 1, p. 194-204, 1975.

JONES, A. B.; DENNISON, W. C.; PERSTON, N. P. Integrated treatment of shrimp effluent by sedimentation, oyster filtration and macroalgal absorption: a laboratory scale study. Aquaculture, Amsterdam, v. 193, n. 1, p. 155-178, 2001.

JONES, A. B.; PERSTON, N. P.; DENNISON, W. C. The efficiency and condition of oysters and macroalgae used as biological filters of shrimp pond effluent. Aquaculture Research, Oxford, v. 33, n. 1, p. 1-19, 2002.

JONES, A. B.; PRESTON, N. P. Oyster filtration of shrimp farm effluent, the effects on water quality. Aquaculture Research, Oxford, v. 30, n. 1, p. 51-57, 1999.

LACERDA, L. D.; SANTOS, J. A.; LOPES, D. V. Fate of copper in intensive shrimp farms: bioaccumulation and deposition in pond sediments. Brazilian Journal of Biology, São Paulo, v. 69, n. 1, p. 851-858, 2009.

LEFEBVRE, S.; BARILlÉ, L.; CLERC, M. Pacific oyster (Crassostrea gigas) feeding responses to a fishfarm effluent. Aquaculture, Amsterdam, v. 187, n. 1-2, p. 185-198, 2001.

MACHADO, I. C.; KOGA, S. M.; WOIOECHOVSKY, E.; GELLI, D. S. Estudo da ocorrência de contaminação orgânica no estuário da Cananéia-SP, Brasil, como subsídio para a extração, manejo e cultivo da ostra do mangue (Crassostrea brasiliana). 2 - Análise da ostra (tecidos moles e líquido intravalvar). Higiene Alimentar, São Paulo, v. 15, n. 83, p. 44-48, 2001.

MADRIGAL, E.; PACHECO, O.; ZAMORA, E.; QUESADA, R.; ALFARO, J. Tasa de filtración del ostión de manglar (Crassostrea rhizophorae, Guilding, 1828), a diferentes salinidades y temperatura. Revista de Biologia Tropical, San Jose, v. 33, n. 1, p. 77-79, 1985.

MINISTÉRIO DA PESCA E AQUICULTURA - MPA.
Boletim estatístico da pesca e aquicultura 2010. Brasília: MPA, 2012. 129 p.

MENDES, P. P.; ALBUQUERQUE, M. L. L. T.; QUEIROZ, D. M.; SANTOS, B. L. S.; LIMA, A. C.; LOPES, Y. V. A. Aclimatação do camarão marinho Litopenaeus vannamei (Boone, 1931) à água doce com diferentes estratégias de alimentação e calagem. Acta Scientiarum. Animal Sciences, Maringá, v. 28, n. 1, p. 89-95, 2006.

RAINBOW, P. S.; AMIARDNTRIQUET, C.; AMIARD, J. C.; SMITH, B. D.; BEST, S. L.; NASSIRI, Y.; LANGSTON, W. J. Trace metal up taker atesin crustaceans (amphipods andcrabs) from coastal site sin NW Europe differential yen riched with trace metals. Marine Ecology Progress Series, Oldendorf, v. 203, n. 1, p. 183-189, 1999.

RAMOS, R.; VINATEA, L.; ANDREATTA, E. R.; COSTA, R. H. R. Tratamento de efluentes de tanques de criação de Litopenaeus vannamei por sedimentação e absorção de nutrientes pela macroalga Ulva fasciata. Boletim do Instituto de Pesca, São Paulo, v. 34, n. 3, p. 345-353, 2008.

RAMOS, R.; VINATEA, L.; COSTA, R. Tratamiento de efluentes del cultivo de Litopenaeus vannamei por sedimentación y filtración por la ostra Crassostrea rhizophorae. Latin American Journal of Aquatic Research, Valparaíso, v. 36, n. 2, p. 235-244, 2008.

RAMOS, R.; VINATEA, L.; SANTOS, J.; COSTA, R. Tratamiento de efluentes del cultivo de Litopenaeus vannamei mediante procesos de sedimentación, filtración y absorción. Latin American Journal of Aquatic Research, Valparaíso, v. 38, n. 2, p. 188-200, 2010.

RAMOS, R.; VINATEA, L.; SEIFFERT, W.; BELTRAME, E.; COSTA, R. Treatment of shrimp effluent by sedimentation and oyster filtration using Crassostrea gigas and C. rhizophorae. Brazilian Archives of Biology and Technology, Curitiba, v. 52, n. 3, p. 775783, 2009.

REYES, L. M. A. Fundamentos de acuicultura marina. Santafé de Bogotá: INPA, 1995. 225 p.

RUPPERT, E. E.; BARNES, D. R. Zoologia dos invertebrados. 6. ed. São Paulo: Rocca, 1996. 1029 p.

SOTO, D.; MENA, G. P. Filter feeding by the freshwater mussel Diplodon chilensis as a biocontrol of salmon farming eutrophication. Aquaculture, Amsterdam, v. 171, n. 1, p. 65-81, 1999.

WARD, J. E. Biodynamics of suspension-feeding in adult bivalve molluscs: particle capture, processing and fate. Invertebrate Biology, Oxford, v. 115, n. 3, p. 218231, 1996. 